\title{
Arbitrary-Oriented Detection of Insulators in Thermal Imagery via Rotation Region Network
}

\author{
Hanbo Zheng, Senior Member, IEEE, Yang Liu, Yonghui Sun, Jinheng Li, Zhen Shi, Chaohai Zhang, \\ Chun Sing Lai, Senior Member, IEEE, and Loi Lei Lai, Life Fellow, IEEE
}

\begin{abstract}
The precise location of insulators in infrared images is of great significance for insulator condition monitoring and fault diagnosis. Due to the characteristics of insulators themselves and the use of handheld infrared cameras, insulators usually appear in infrared images with different aspect ratios and main axis orientations. Therefore, it is very important and necessary to make full use of the prior knowledge of the insulator itself to accurately locate it. However, most of the existing methods use axial horizontal detection boxes to detect insulators, which cannot take into account the characteristics of the insulator well. When there are large overlapping areas of two horizontal detection boxes, the non-maximum suppression algorithm may lead to missed detection of the object. In order to further improve the accuracy of the detection algorithm, this paper makes full use of the prior features carried by the insulator itself, and optimizes Faster R-CNN from five aspects: rectangular box representation, feature extraction, candidate box generation, anchor design, and feature alignment. An oriented detection model for infrared images of insulators is constructed. Comparative experiments with a variety of mainstream detection methods were carried out on the constructed infrared dataset. The results show that the proposed method is superior to other models in detection accuracy. When the intersection and union ratio is 0.5 , the average precision reaches $95.08 \%$. In addition, it can also effectively predict the shape and angle information of insulators in complex scenes, laying a beneficial foundation for subsequent diagnosis automation tasks.
\end{abstract}

Index Terms-Convolutional Neural Network, Orientation detection, Infrared image, Insulator, prior knowledge

\section{INTRODUCTION}

$\mathrm{W}$ ITH the development of power system, the number of transmission lines and substations is increased, and the

This work was supported in part by the National Natural Science Foundation of China under Grant 51907034, and in part by the Specific Research Project of Guangxi for Research Bases and Talents under Grant 2020AC19010. (Corresponding authors: Chun Sing Lai and Loi Lei Lai.)

Hanbo Zheng, Yang Liu, Yonghui Sun, Jinheng Li, Zhen Shi, Chaohai Zhang are with the Guangxi Key Laboratory of Intelligent Control and Maintenance of Power Equipment, Guangxi University, Nanning 53004, China (e-mail: $\quad$ hbzheng@gxu.edu.cn; $\quad 1812391036 @$ st.gxu.edu.cn; 1912301049@st.gxu.edu.cn; 1812301016@st.gxu.edu.cn; eee0619@sina.com; chzhang@gxu.edu.cn).

Chun Sing Lai is with the Brunel Interdisciplinary Power Systems Research Centre, Department of Electronic and Electrical Engineering, Brunel University London, UB8 3PH London, U.K., and also with the Department of Electrical Engineering, School of Automation, Guangdong University of Technology, Guangzhou 510006, China (e-mail: chunsing.lai@brunel.ac.uk).

Loi Lei Lai is with the Department of Electrical Engineering, School of Automation, Guangdong University of Technology, Guangzhou 510006 , China (e-mail: 1.1.lai@ieee.org). corresponding number of insulators is also increased rapidly. Considered as one of the power equipment that plays the function of electrical insulation and mechanical support, the insulator plays an important part in the safe operation of the power system [1]-[3]. The failure of the insulator will cause short circuits between conductors. It can also severely damage electrical equipment and cause unplanned power outages of the power grids. According to a large number of experiments available in the literature, the main cause of insulator failure is attributed to abnormal temperature. Therefore, monitoring the thermal condition of insulators is often the focus of electrical power engineers in regular inspection tasks. Infrared thermography (IRT) has the advantages of large detection range, fast detection speed, non-contact, no electromagnetic interference, etc., and is widely used in the detection of the thermal state of insulators [4]. However, the massive infrared images obtained through infrared cameras still require a large number of engineers to carry out analysis and judgement. This procedure is not only time-consuming but also inefficient [5]-[7]. In recent years, inspection robots equipped with infrared cameras have begun to be adopted by power companies, which has improved the automation level of power inspections [8]-[9]. The automatic detection technology based on computer vision can greatly reduce the discrimination time and effectively improved the inspection efficiency, which has become a research hotspot in the field of insulator inspection.

Among them, detection method based on deep learning is an end-to-end learning method. It can learn complex advanced feature representations from large amounts of original data through multi-layer network stacking [10]. As AlexNet won the ImageNet image classification competition, researchers around the world began to apply deep learning techniques to other areas of computer vision, such as image retrieval and image segmentation. At the same time, a large number of general detectors based on deep learning, such as R-CNN [11] and Fast R-CNN [12], are designed in the field of target detection. Because the universal detector has strong generalization ability and adaptability, researchers engaged in power engineering vision have carried out researches on the automatic detection of insulators based on the universal detector, and achieved many satisfactory results.

However, many current insulator detection methods are just simple migration applications of general object detectors in the power field. General object detectors are mature and accurate enough to detect the most common objects, such as people or cars. However, when detecting elongated objects with different orientations, such as insulators, it is difficult to take into account the orientation and shape characteristics of the object. 
When there are complicated situations such as tilt and mutual occlusion, the detection accuracy and effect are not satisfactory. In addition, the detection box output by the general detector is a horizontal rectangular box, and its location and identification of the insulator may also cause unnecessary background noise and object overlap. In order to solve the above problems, this study makes full use of the prior knowledges carried by the insulator itself, and proposes a high-precision oriented detection method for infrared insulators.

The main contributions of this paper are as follows:

1) A high-precision oriented detection method for infrared images of insulators based on deep learning is proposed. Combining the rotating detection box with the deep learning method effectively solves the defect so that the output detection box of the non-oriented detection method is easily deleted by the non-maximum value suppression algorithm and reduces the detection accuracy.

2) The improved residual network and feature pyramid are embedded in the proposed model. These two modules can not only make full use of feature information and reduce the amount of calculation, but also can effectively improve the multi-scale detection capability of the model.

3) The rotation region proposal network (RRPN) is embedded in the proposed model. This module enables the model to generate high-quality rotatiing candidate regions containing potential targets.

4) Aiming at the geometric characteristics of insulators, an adaptive method of anchor combining improved clustering algorithm and statistical analysis is proposed. This method allows the model to distinguish between targets with similar backgrounds well.

5) The rotation region of interest (RRoI) pooling layer is introduced into the proposed model. This module can effectively match the candidate region with the feature matrix, retain features that are beneficial to the detection task, suppress features that are not helpful to the task.

The remainder of this paper is organized as follows. Section II analyzes the research background and related work of this study, and reveals the deficiencies of non-oriented detection methods through an example. Section III gives a detailed introduction and explanation of the proposed model and its various improvement modules. In Section IV, we describe the experimental process and evaluate and analyze the results. Finally, conclusions and prospects are presented in Section V.

\section{RELATED WORK}

In the past ten years, many scholars have carried out work on automatic detection methods for insulators and have achieved some results. According to the different feature extraction methods, the detection methods of power equipment can be divided into artificial design features and deep learning methods[13]. Table I summarizes some of the latest research results in the application of the above two types of methods in the field of power equipment automation in recent years. The content mainly includes the detection accuracy and speed evaluation of their methods. Among them, the artificial design feature methods are mainly realized through the combination of manual features and traditional machine learning. Reference [14] proposed an insulator detection method combining the original convex energy function and the active contour. However, this method requires a large amount of calculation, and the hyperparameters in the energy function need to be manually set. In [15], an insulator detection method combining Sobel operator and accelerated robust feature operator was proposed. However, this method has a cumbersome detection process. Zhao et al. [16] combined orientation angle detection and binary shape features to locate and identify insulators. But this method is only suitable for overhanging insulators with a larger size, and the detection time is longer. Reference [17] proposed a feature operator that combines rotation invariance and local orientation mode to represent the insulator, and uses the feature vector constructed by the operator to train the SVM for completing the insulator identification and location process. The above-mentioned feature extraction methods are manually designed according to specific objects, and are limited to low-level feature representation. They do not have the learning ability to improve the recognition accuracy as the number of samples increases. In addition, their effectiveness and reliability are suppressed by the shooting angle and distance.

With the continuous development of deep learning in the field of computer vision, image recognition technology based on deep learning can greatly reduce the detection time for power equipment, and effectively improve the accuracy and efficiency of equipment condition detection. Wang et al. [18] proposed a lightweight $\mathrm{CNN}$ to identify ice-coated insulators on edge intelligent terminals. This method uses MobileNetv 3 as the feature extraction network, and SSD [19] as the identification network for insulator ice thickness measurement. It requires small memory, fast calculation speed, and strong adaptability to edge computing terminals with limited computing resources. In [20], a new type of deep CNN with a cascade structure is used to solve the two-level problem of insulator location and defect detection. It can successfully detect insulator defects under various conditions and meet the robustness and accuracy requirements of insulator defect detection, but the data-driven detection method lacks generalization in insulator defect detection in aerial images. Reference [21] improved the region generation network and NMS method of Faster R-CNN [22] to detect insulators. The improved method significantly improves the detection effect of insulators with different aspect ratios and different scales, and can also effectively detect insulators that are shielded from each other. However, the detection speed as an important evaluation index has not been compared and tested. Reference [2] proposed an improved FSSD detection model for infrared images of substation insulators. However, this model cannot improve the detection accuracy of the model while maintaining a high detection speed. Liu et al. [23] proposed an automatic location identification and diagnosis method for external power insulation equipment based on YOLOv3. However, the original YOLOv3 model has poor detection performance for large and medium-scale objects, and has no obvious advantages in the location and recognition of power equipment. 
The above deep learning detection methods are referred to as a non-oriented detection method in this paper. They all use the horizontal detection bounding box to detect objects, and have good detection effects when detecting general objects in natural scenes. However, the task of insulator detection is different from general object detection. Due to the shooting angle, the characteristics of the insulator itself, and the voltage level, the insulator will appear in the infrared image in different overhead modes. When insulators with different orientations are cross occlusion or erected in a parallel connection manner, two horizontal detection boxes surrounding different insulator strings will overlap and cause an excessively large overlap area. At this time, if the existing non-oriented detection algorithm is used to detect these inclined insulators, the output horizontal detection box can easily be suppressed and deleted by NMS, which leads to missed detection of the insulator and reduces the detection accuracy. The test results are shown in Fig. 1. It can be seen that due to NMS, the insulator strings A, C, and F in Test 1 are not detected, and the blocked insulator string $\mathrm{A}$ in Test 2 is deleted. Therefore, the non-oriented detection method is not the best method for detecting objects with arbitrary orientations, relatively large width and height, such as insulator strings.
To solve the problem that non-oriented detection methods cannot effectively detect multi-oriented objects and improve the detection accuracy of detection algorithms, experts in different fields have proposed oriented detection methods based on rotating rectangular boxes [24-25]. Inspired by the above oriented detection ideas, researchers have also carried out work on the oriented detection methods for power equipment. Li et al. [26] proposed an insulator orientation recognition method based on improved SSD. This method can well complete the detection of inclined insulators, but the
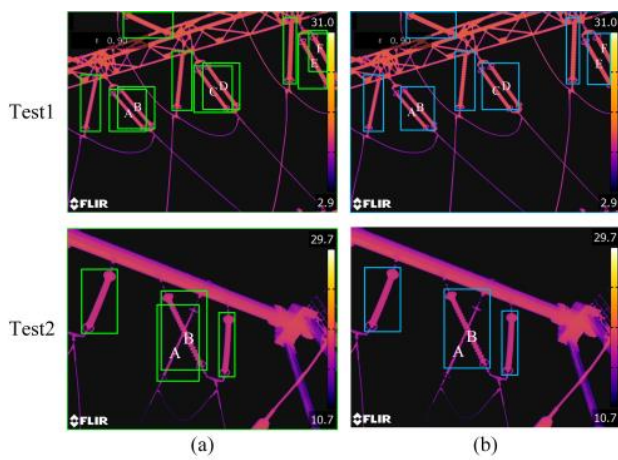

(b)

Fig. 1. (a) Ground truth box. (b) Detection result.

TABLE I

LITERATURE REVIEW OF RELATED WORK

\begin{tabular}{llll}
\hline \hline $\begin{array}{c}\text { Detection } \\
\text { methods }\end{array}$ & Detection accuracy & Detection speed & $\begin{array}{l}\text { location } \\
\text { strategy }\end{array}$ \\
\hline
\end{tabular}

14 can effectively segment insulators with uneven texture from aerial images. The missed detection rate in 100 aerial test images is only 0.05 , and the detection accuracy is high

[15] It can accurately identify the insulators of the railway catenary from the target image

The recognition accuracy is higher than most other deep learning methods on the self-built dataset, and the mAP is as high as 0.75

The detection accuracy and recall rate are as high as 0.91 and

[20] 0.96 on the self-built dataset, which meets the detection accuracy requirements of related scenes

Compared with the original model, the detection accuracy on the self-built dataset has been greatly improved, and its average accuracy index has reached 0.82

In the self-built dataset, the detection accuracy is slightly

[2] higher than the original model, and the average accuracy is increased by 0.72

23] On the self-constructed dataset, its average precision average index is as high as 0.89

[26] On the self-built insulator visible light dataset, its average accuracy reached 0.82

The average accuracy of the self-built power equipment infrared dataset is as high as 0.94
On the self-built power equipment infrared dataset, its average accuracy is as high as 0.93
This method requires a lot of calculations, and it takes $51 \mathrm{~s}$ to detect a picture on average, which cannot be applied to field detection

This method has a small amount of calculation, and the detection time in the sample test picture is only $0.08 \mathrm{~s}$

This method has a small amount of calculation. The average detection time in the sample testing for a picture is $2.66 \mathrm{~s}$, and the detection speed needs to be further improved

This method requires small memory, fast calculation speed, up to $30 \mathrm{fps}$, and has strong adaptability under edge computing terminals with limited computing resources.

The detection speed is $0.36 \mathrm{~s}$ per picture, which can be applied to on-site detection requirements where real-time requirements are not strong

Two-stage detection method, the detection speed is slow

The detection speed is faster, with a fps of 23.81 , which is slightly slower than the original model, and basically meets the requirements of real-time detection

The detection speed is very fast, the fps is up to 55, which meets the real-time detection requirements

The FPS is 20, and the detection speed is slower than the original model

Need to train two independent networks separately, the detection process is complicated and cumbersome, and the detection speed is very slow
Segmentation

Feature point matching + horizontal box

Rotating box

Texture matching + horizontal box

Horizontal box

Horizontal box

Horizontal box

Horizontal box

Horizontal box

Rotating box

Rotating box

Rotating box 
detection accuracy of this method is low, and there are a large number of falsely detected insulator string samples. Reference [27] proposed a single-stage oriented detection method for infrared images of power equipment. However, this method can only detect the equipment components in the same orientation in the infrared image, and cannot complete the detection task of the insulator orientations arranged in any main axis orientation in the actual scene. To further improve the detection accuracy of the orientation detection algorithm, Lin $e t$ al. [28] proposed a combination of space transformer network and Faster R-CNN infrared image orientation detection method for power equipment. This method achieves better detection accuracy in the detection of four types of power equipment components, but two independent networks need to be trained separately. The detection process is complicated and cumbersome, requiring repeated debugging and parameter adjustment.

Therefore, in order to achieve effective oriented detection of insulators in infrared images, the proposed method in this paper combines the prior information carried by the insulator itself, which effectively solves the defects of non-oriented detection methods, while ensuring accuracy, it can further increase the recall and reduce the number of missed inspections.

\section{THE PROPOSED METHOD}

\section{A. Overall Framework}

We present the details of the detection pipeline of the method proposed in this paper, which is illustrated in Fig. 2. Specifically, it contains two main stages, one to complete the rough regression of the rotating prior box and the other to complete the refinement of the proposals. In this first stage, We use the improved residual network (ResNet) and feature pyramid network (FPN) structures [29]-[30] to get the feature maps of three feature levels, namely $\{$ P3, P4, P5 $\}$. Given the multiscale feature maps, the RRPN completes the first regression through back propagation and generates the rotated proposals on the original image that may contain insulator target. In the second stage, the RRoI pooling layer performs uniform scaling for each rotated proposal of different sizes. Then, these feature matrices will be sent to the fully connected neural network for another regression, and the final detection box close to the insulator target is generated.

\section{B. Improved ResNet Architecture}

The residual network based on the residual architecture is a feature extraction network widely used in deep learning [29]. A residual backbone consists of an input stem $\mathrm{C} 1$ and four consecutive residual stages $\mathrm{C} 2-\mathrm{C} 5$, which is illustrated in Fig. 2. Among them, a stage module includes a down-sampling block and several identity mapping blocks. In the original version of Caffe implementation, the input stem consists of a convolution kernel with a size of $7 \times 7$ and a pooling layer with a size of $3 \times 3$. In the residual block of each stage, $1 \times 1$ convolution with a stride of 2 completes the downsampling of the feature map, as shown in Fig. 3(a). However, there are two drawbacks in the original implementation. First of all, $7 \times 7$ convolution requires more parameters and higher computational cost. Then, the $1 \times 1$ convolution with a stride of 2 in path $B$ of the residual block loses a large amount of feature map information, which results in a decrease in the detection performance of the model.

Based on the above analysis, we make two improvements to the original version of the residual network. First, we switch the size of the stride of the first two convolutions in path A of the down-sampling block. Second, we replace the $7 \times 7$ convolution in the input stem with three consecutive $3 \times 3$ convolutions. The modified result is shown in Fig. 3(b).
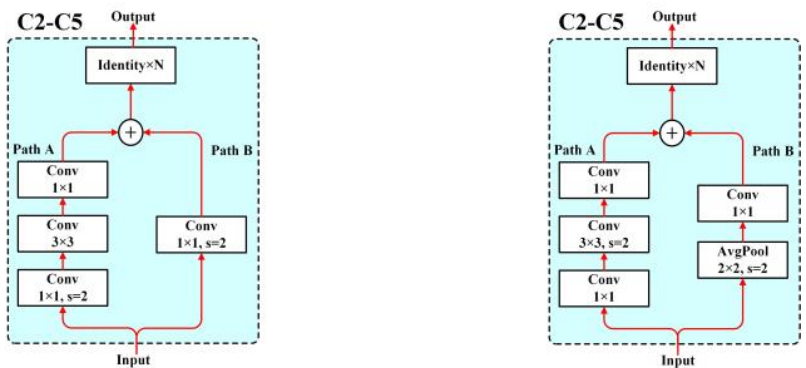

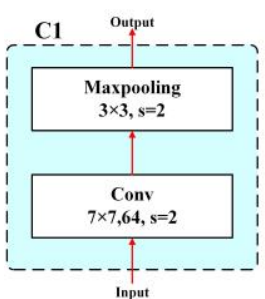

(a)

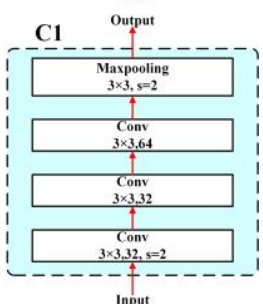

(b)
Fig. 3. Comparison of two residual network structures (a) original version. (b) improved version.

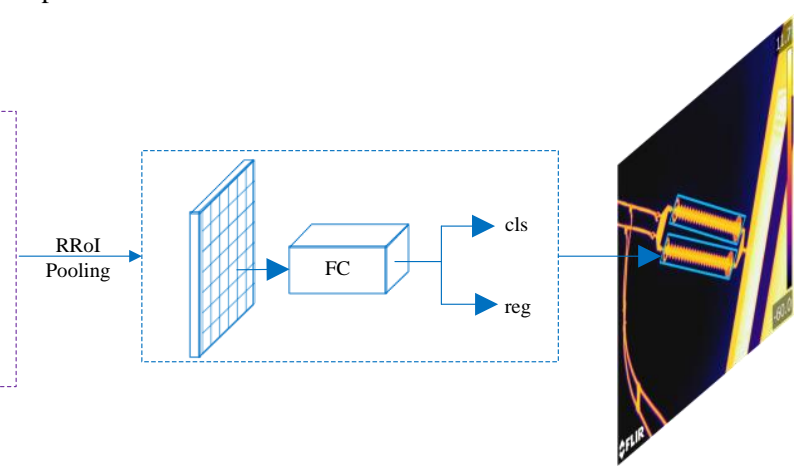

Fig. 2. Detection pipeline of the proposed method. 


\section{Orientation Detection Box Representation}

Non-oriented detection tasks are usually marked with axis-aligned horizontal detection boxes. These boxes are usually uniquely determined by the coordinates of the upper left corner and the lower right corner. To meet the needs of detection task, the above parameters usually need to be converted into the four tuples, namely $\left(x_{c}, y_{c}, w, h\right)$. Among them, $\left(x_{c}, y_{c}\right)$ represent the coordinates of the center point, $w$ denotes the width of the box, and $h$ denotes the height of the box. The labeling parameters are shown in Fig. 4(a)

In the orientation detection method adopted in this paper, we need to add a parameter that records the angle information to label the insulator target in any orientation. Specifically, we add the angle parameter theta based on the 4 parameters of the original horizontal box to determine an orientation detection box $\left(x_{c}, y_{c}, w, h, \theta\right)$. The parameter $\theta$ represents the inclination angle of the insulators, which is defined as the angle between the boundary detection box and the positive half axis of the $\mathrm{x}$-axis. In addition, we specify that the anti-clockwise rotation of the angle is positive, and the angle range is $\left[0,180^{\circ}\right)$. The labeling parameters are shown in Fig. 4(b).

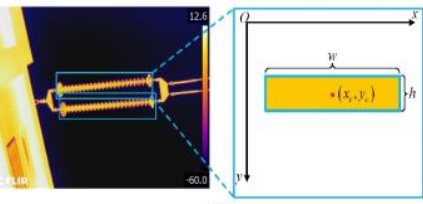

(a)

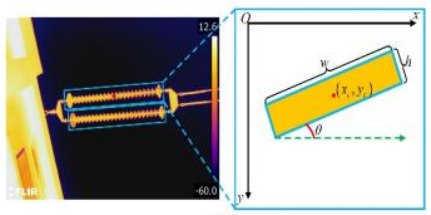

(b)
Fig. 4. Comparison of parameters used in horizontal and orientation detection boxes. (a) Definition of a horizontal bounding box. (b) Definition of a rotated bounding box.

\section{Rotation Region Proposal Network}

Different from the region proposal network (RPN) of the Faster R-CNN, rotation region proposal network (RRPN) is a kind of convolutional neural network that can generate the rotating proposals that may contain insulator regions through learning. The entire network architecture is depicted in Fig. 5. Among them, the cls layer is the classification layer, which is used to predict whether the object is a background or an insulator; the reg layer is a regression layer, which is used to predict the location area of the insulator. As described in the figure, the network firstly extracts deeper features from the feature maps obtained from the feature pyramid network

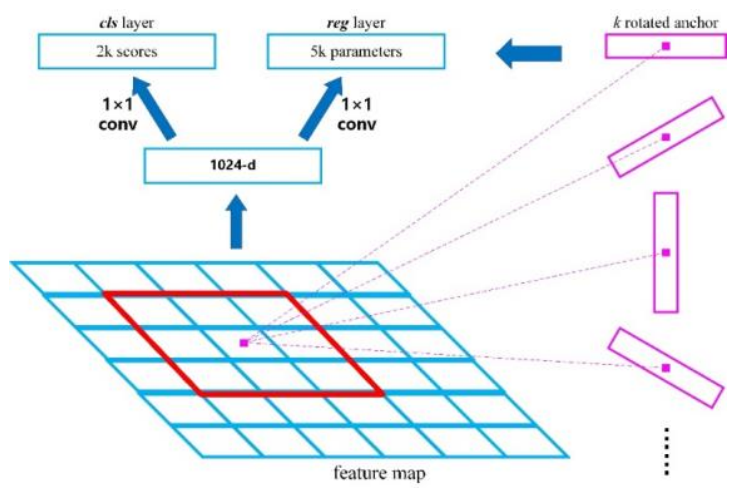

Fig. 5. Diagram of the rotation region proposal network. through a $3 \times 3$ convolution and generates $\mathrm{k}$ rotated prior boxes at each position of the map. Then, the extracted high-dimensional vectors are sent into the cls layer and the reg layer through two $1 \times 1$ convolutions, and the rotated region of interest is obtained by learning the classification and location regression of the object.

\section{E. Rotation Anchor Generation}

The traditional RPN in Faster R-CNN uses anchors that only use scale factor and aspect ratio parameters to generate prior boxes for subsequent network training. This axis-aligned anchor strategy cannot cover all shapes of strip-like insulators in different orientations. As earlier discussed, it is difficult to solve the defect detection problem of insulators. To better improve the detection accuracy of the insulator string and fit the insulator contour, we adjust the rotation prior box according to the true shape of the insulator target. The anchor selection strategy is shown in Fig. 6. First, an angle parameter is added based on the first two parameters to control the orientation of a prior box, which is set as $\left\{0^{\circ}, 30^{\circ}, 60^{\circ}, 90^{\circ}, 120^{\circ}, 150^{\circ}\right\}$ and is shown in Fig. 6(a). Second, the aspect ratio is modified according to the actual distribution of the insulator target, which is shown in Fig. 6(b). Besides, the scale of the rotate box is set as $\{8,16,32\}$, which is shown in Fig. 6(c). The selection strategy will be explained in more detail in Section IV. D.

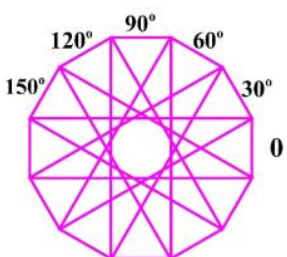

(a)

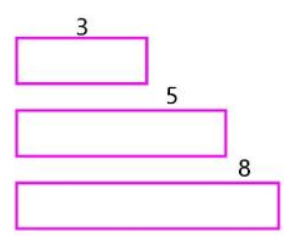

(b)

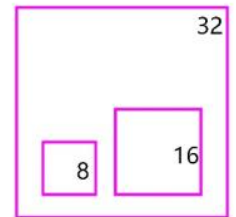

(c)
Fig. 6. The generation strategy of rotating anchor.

\section{F. Rotating Intersection-over-Union Computation of Rotated Box}

The Intersection-over-Union (IoU) is a similarity index commonly used in the research field of target detection, which is widely used in the selection of positive samples in the training stage and selection of the final detection results in the testing phase. In the classical non-oriented detection task, the intersection and union of two horizontal rectangular boxes can be obtained by the simple four calculation of the area of the horizontal rectangular box, as shown in Fig. 7(a). However, in the orientation detection task with angle parameters, the classical calculation method of IoU is not applicable. This is because the rotation rectangle box can be generated in any orientation and the intersection of the two boxes is a convex polygon. Rotating Intersection-over-Union (RIoU) calculation method for the rotating box was designed to solve this problem. The geometric principle of the method is shown in Fig. 7(b). It can be seen from the figure that the basic idea of this method is to decompose a convex polygon into several triangles. By calculating the area of each triangle, the intersection of convex 


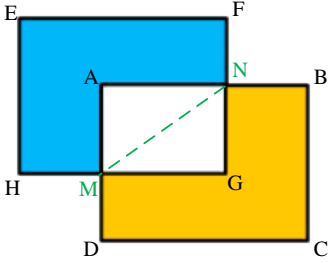

(a) Intersection of Union

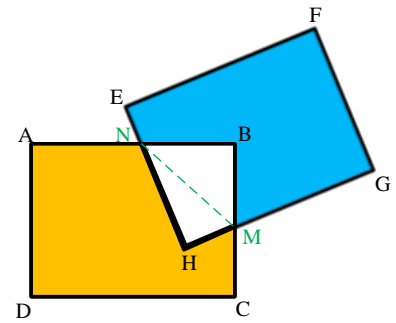

(b) Rotating Intersection of Union
Fig. 7. Geometric principle of IoU and RIoU.

polygons can be obtained by adding the area of the multiple triangles. For IoU and RIoU, the corresponding definitions are as follows:

$$
\begin{gathered}
I o U=\frac{\operatorname{Area}(A B C D \cap E F G H)}{\operatorname{Area}(A B C D \cup E F G H)}=\frac{S_{A N G M}}{S_{A B C D}+S_{E F G H}-S_{A N G M}} \\
R I o U=\frac{\operatorname{Area}(A B C D \cap E F G H)}{\operatorname{Area}(A B C D \cup E F G H)}=\frac{S_{N B M}+S_{N M H}}{S_{A B C D}+S_{E F G H}-S_{N B M}-S_{N M H}}
\end{gathered}
$$

Among them, $S_{A N G M}, S_{A B C D}, S_{E F G H}, S_{N B M}$, and $S_{N M H}$ represent the area of each polygon in Fig. 7.

\section{G. Training strategy of RRPN}

The generated rotated prior boxes were divided into positive samples and negative samples to train the RRPN. This was done following the calculation result of RIoU and the constraint of the angle between the rotating anchor. The rotation anchor that complies with the following principles is determined as a positive sample: 1) the highest RIoU overlap with some real labeled boxes and 2) the RIoU overlap with any real labeled boxes greater than 0.7 and their angle error is less than 15 degrees. The negative samples are characterized by the following: 1) the RIoU overlap with all real labeled boxes less than 0.3 or 2 ) the RIoU overlaps with any real labeled boxes larger than 0.7 but their angle error is larger than 15 degrees. Rotating prior boxes that are neither positive nor negative samples are not used as training samples of RRPN.

Based on the above sampling strategy, we use a multi-task loss function to train RRPN, which is defined as:

$$
L_{R R P N}=L_{c l s}+\lambda b L_{r e g}
$$

where $L_{c l s}$ is classification loss and $L_{r e g}$ is regression loss of the rotated anchor. $b$ represents the category label. When it is equal to 1 , the detected object is the foreground, and when it is equal to 0 , the object is the background. $\lambda$ represents the balance coefficient, which is used to adjust the weight of the two tasks in the total task.

For $L_{c l s}$, the cross-entropy loss is adopted and is defined as:

$$
L_{c l s}(p, b)=-\sum_{i \in \text { Pos }}^{N} b \cdot \log \left(p_{1}\right)-\sum_{i \in \text { Neg }}^{N}(1-b) \cdot \log \left(p_{0}\right)
$$

where $b$ is a two-category label, equal to 0 or $1 . p_{l}$ represents the probability that the object in the detection box belongs to the insulator string and $p_{0}$ represents the probability of being the background.

For $L_{r e g}$, the rotating prior boxes identified as the negative sample was ignored. The smooth ${ }_{L l}$ loss function to carry out regression training for the rotating anchor identified as positive samples was used. It is defined as:

$$
L_{\text {reg }}\left(t^{*}, t\right)=\sum_{i \in \text { Pos }}^{N} \sum_{i \in\left\{x_{c}, y_{c}, w, h, \theta\right\}} \text { smooth }_{L_{1}}\left(t_{i}^{*}-t_{i}\right)
$$

where $\left(x_{c}, y_{c}, w, h, \theta\right)$ denotes the center coordinate, width, height, and rotation angle, respectively. $t=\left(t_{x}, t_{y}, t_{h}, t_{w}, t_{\theta}\right)$ denotes the error tuple of the regression prediction boxes and the rotated prior boxes. $t^{*}=\left(t_{x}{ }^{*}, t_{y}{ }^{*}, t_{h}{ }^{*}, t_{w}{ }^{*}, t_{\theta}{ }^{*}\right)$ represents the error tuple of the ground truth boxes and the rotated prior boxes.

Furthermore, Loss $\operatorname{smooth}_{L I}(x)$ represents the smoothed loss based on the L1 norm, which is defined as follows:

$$
\operatorname{smooth}_{L_{1}}(x)= \begin{cases}0.5 x^{2} & \text { if }|x|<1 \\ |x|-0.5 & \text { otherwise }\end{cases}
$$

The elements contained in the tuple are defined as follows:

$$
\begin{aligned}
& \left\{\begin{array}{l}
t_{x}=\frac{x-x_{a}}{w_{a}}, t_{y}=\frac{y-y_{a}}{h_{a}} \\
t_{h}=\log \frac{h}{h_{a}}, t_{w}=\log \frac{w}{w_{a}} \\
t_{\theta}=\theta-\theta_{a}+k \pi, k \in Z
\end{array}\right. \\
& \left\{\begin{array}{l}
t_{x}^{*}=\frac{x^{*}-x_{a}}{w_{a}}, t_{y}^{*}=\frac{y^{*}-y_{a}}{h_{a}} \\
t_{h}^{*}=\log \frac{h^{*}}{h_{a}}, t_{w}^{*}=\log \frac{w^{*}}{w_{a}} \\
t_{\theta}^{*}=\theta^{*}-\theta_{a}+k \pi, k \in Z
\end{array}\right.
\end{aligned}
$$

where $x, x_{a}$, and $x^{*}$ denote the $\mathrm{x}$-coordinates in the regression output result, rotating prior detection box, and ground truth box, respectively. The remaining few elements are similar to the aforementioned $x$ and will not be described in detail. The range of parameters $t_{\theta}$ and $t_{\theta}{ }^{*}$ should always comply with the angle constraint in the range of $\left[0,180^{\circ}\right)$.

\section{H. Rotated Region of Interest Pooling layer}

The suggested method in this paper needs to use the full connection layer to realize the final classification and regression of objects. As a result, the multi-scale rotated feature maps derived from the map of the rotated region of interest (RRoI) generated by RRPN must be normalized before they are input to the layers. RoI Pooling is a basic pooling operation in Faster R-CNN, which can extract fixed-size feature matrix from the region of interest of different sizes. It plays an important part in non-oriented detection tasks based on axis align horizontal rectangular boxes. However, it cannot comply with the task of refinement detection of insulators with the complicated background interference, which leads the poor performance in the subsequent classification and location task. Therefore, the RRoI Pooling Layer is adopted to better handle the RRoI extracted from the first stage of RRPN. The operation diagram is shown in Fig. 8. First, the feature map corresponding 


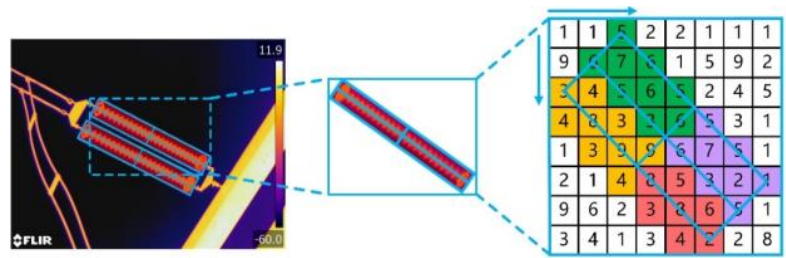

Fig. 8. RRoI Pooling Layer.

to the rotation candidate area will be divided into many cells with the same area according to the input parameters. Each bin has the same orientation as that of the proposal. Then, each rotation sub-region is traversed, and the maximum pooling is carried out for each rotating region. Compared with the RoI-Pooling layer, RRoI Pooling performs uniform scaling for each rotated proposal of different sizes, aspect ratios, and angles.

\section{EXPERIMENTS}

\section{A. Dataset}

Due to the particularity of data security in the power industry, there is currently no public power equipment infrared image dataset for research and comparison. Therefore, in order to evaluate the proposed method, it is necessary to construct an infrared image dataset of insulators. The dataset consists of data that is expanded and preprocessed from the original infrared images. Among them, the original infrared images are obtained by electric power inspectors from multiple substations of different voltage levels using infrared cameras to take pictures of the insulators on site. Specifically, the inspectors use the T640 and T660 infrared cameras developed by FLIR to collect on-site images. The average distance between the camera and the insulator is about $2 \sim 4 \mathrm{~m}$, and the shooting scene includes $110 \mathrm{kV}$ and $220 \mathrm{kV}$ multiple substations. After deleting some unclear and low-contrast images, the original data for infrared image of the insulator in this paper is obtained. Among them, the dataset contains a total of 2760 infrared images of insulators, and a total of 8410 insulator strings are marked. Both the training set and the test set are randomly shuffled, with a ratio of $8: 2$.

\section{B. Experimental Setup}

The proposed method in this paper was implemented using the deep learning framework based on the Pytorch 1.1.0. Among them, the hardware environment in this study is based on the Ubuntu 16.04 server platform with an Intel Xeon W-214 CPU and an Nvidia GeForce RTX 2080Ti with 12 GB of memory. In the pre-processing stage, the dimensions of all original images were uniformly scaled to $640 \times 640$ to adapt to the network input and the insulators in all images were labeled with rlabelImg software. Then, $80 \%$ of the images were randomly selected in the constructed dataset for training and the remaining images as the test set. To avoid overfitting and improve the recognition ability of the network, we randomly flipped the images of the training set horizontally and transformed the hue, saturation, and exposure with a probability of $50 \%$. The improved ResNet50 was utilized as the backbone of the oriented detection task. The initial weight of the network adopted the pretraining weights downloaded from Github for ImageNet classification. The chosen optimizer was the SGD optimizer with a momentum of 0.9. The network is trained for $15 \mathrm{k}$ iterations in total, and 4 pictures are passed in each cycle. In the first 11,000 cycles, we set the learning rate to 0.01 . Then, in the next 2500 cycles and the remaining 1500 cycles, the learning rate is adjusted with a decay of $10 \%$.

\section{Evaluation Metrics}

In order to quantitatively evaluate the detection effect of the method in this paper, we introduced four indicators: precision, recall, F-score, and average precision to reflect the performance of the network.

\section{1) Recall and precision}

The precision rate represents the ratio of the output results by the detection network that are truly treated as positive samples. The recall rate represents the ratio of the detection results output by the detection network and the real labeled boxes that are correctly matched. Both indicators can be calculated from the true-positive (TP), false-positive (FP), and false-negative $(\mathrm{FN})$, which are defined respectively as follows:

$$
\begin{aligned}
& \text { Precision }=\frac{\mathrm{TP}}{\mathrm{TP}+\mathrm{FP}} \\
& \text { Recall }=\frac{\mathrm{TP}}{\mathrm{TP}+\mathrm{FN}}
\end{aligned}
$$

where TP denotes the number of insulator targets judged by the model as positive samples, FP represents the number that the model judges the background as the insulator target and FN represents the number of targets missed by the model.

\section{2) Average precision (AP)}

In the object detection task, the AP reflects the average precision rate in the case of different recall rates. This indicator can be obtained by calculating the area enclosed by the P-R curve, horizontal axis, and vertical axis. $\mathrm{AP}_{50}$ is one of the commonly used metrics, which denotes the AP value when the IoU threshold is $50 \%$. We use $\mathrm{AP}_{50}$ to evaluate the detection performance of our method in this paper.

\section{3) F-Score}

The F-score is a comprehensive evaluation index in object detection. It combines two metrics of precision and recall rate into a single measurement index, which is defined as:

$$
F=\frac{2 \times \text { Precision } \times \text { Recall }}{\text { Precision }+ \text { Recall }}
$$

\section{Selection Strategy of Rotation Anchor Parameters}

The nine anchors are generated based on three sizes $\{128$, $256,512\}$ and three ratios $\{1: 1,1: 2,2: 1\}$ in RPN of the Faster R-CNN. The image size and target size in PASCAL VOC [31] are relatively large, the original anchor size is suitable for the dataset, and good experimental results can be obtained. However, the insulators vary in angle and appearance in our dataset, the original parameter configuration of anchor in RPN did not satisfy our dataset requirements. Thus, it is necessary to modify it to better fit the contour of the target and improve the 


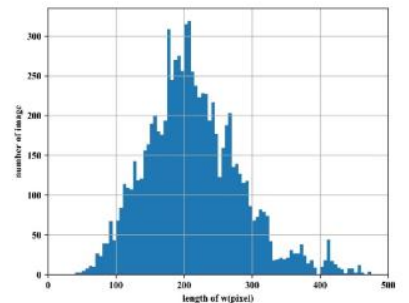

(a)

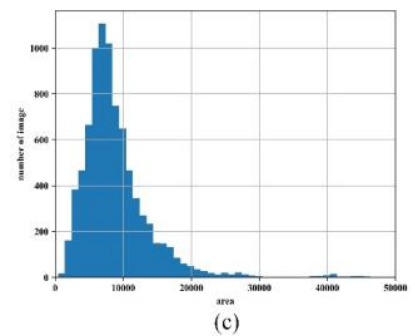

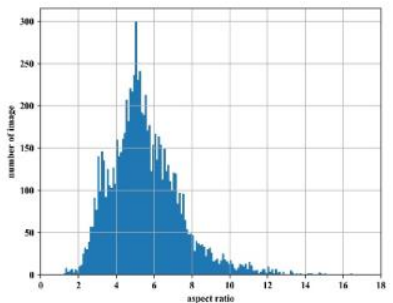

(b)

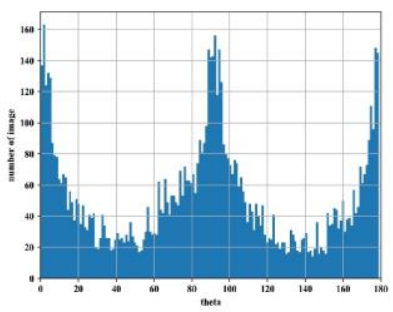

(d)
Fig. 9. Histogram of the statistical distribution of target attributes in the dataset. (a) distribution of long side w. (b) distribution of aspect ratio w/h. (c) distribution of area. (d) distribution of angle.

detection accuracy of the insulator string.

The insulator features in the dataset were analyzed and the main feature distribution of the detection target was plotted in Fig. 9. As can be seen in subfigure (a), the main distribution range of the long side $w$ is about $[125,275]$. The maximum is about 460 pixels and the mean length is 213 . In subfigure (b), the aspect ratio is mainly distributed between 3 and 8 . In subfigure (c), the area is distributed mostly between 3600 and 14000. And in subfigure (d), the inclination angle of the sample is distributed at various angles in a form that approximately satisfies the normal distribution.

After a detailed analysis of the sample attributes in the dataset, we modified the horizontal anchor to a rotating anchor, and performed K-means++ clustering on the dataset labels. The conversion equation of $\mathrm{K}$-means++ is as follows:

$$
d(g t, k)=1-\operatorname{IOU}(g t, k)
$$

where $I O U$ is the intersection over union of ground truth and cluster center rectangular box, $k$ represents cluster center rectangular box, $g t$ represents the ground truth. The results are shown in Table II. The anchor parameters required by the algorithm are obtained through equal area conversion and feature map mapping: the aspect ratio is set to $\{1: 3,1: 5,1: 8\}$, the anchor size is set to $\{8,16,32\}$, and the orientation angle parameter is set as $\left\{0^{\circ}, 30^{\circ}, 60^{\circ}, 90^{\circ}, 120^{\circ}, 150^{\circ}\right\}$.

TABLE II

K-MEANS++ CLUSTERING RESULT

\begin{tabular}{cccc}
\hline \hline Small object & $(100,31)$ & $(143,36)$ & $(178,38)$ \\
\hline Medium object & $(206,38)$ & $(235,38)$ & $(268,42)$ \\
\hline Large object & $(306,49)$ & $(364,51)$ & $(424,69)$ \\
\hline \hline
\end{tabular}

\section{E. Ablation Study}

To evaluate the role played by different modules in the entire detection task, we conducted some ablation experiments on them.

\section{1) Effect of network improvement}

We evaluate the original version and the improved version of
ResNet50 described in Section III. The experimental results of different backbone networks are presented in Table III. Among them, Res50_a and Res50_b represent the original version and improved version of the residual network with fifty layers, respectively. As can be observed from the table that the detection performance of the improved version is slightly better than the original version. It improves F-score by around $0.8 \%$ compared to the original implementation. The improvement of detection performance benefits from the maximum utilization of feature map information.

TABLE III

COMPARISON OF DIFFERENT RESIDUAL NETWORKS

\begin{tabular}{cccc}
\hline \hline Model & Precision $(\%)$ & Recall(\%) & $\mathrm{F}(\%)$ \\
\hline Res50_a & 94.23 & 97.78 & 95.97 \\
Res50_b & $\mathbf{9 5 . 5 6}$ & $\mathbf{9 8 . 0 0}$ & $\mathbf{9 6 . 7 6}$ \\
\hline \hline
\end{tabular}

\section{2) Effect of the RRoI Pooling}

To evaluate the performance of the RRoI Pooling method in the orientation detection task, we conducted an ablative study, and the experimental results are given in Table IV. As expected, using the RoI Pooling operation in our model has poor performance. This pooling operation will introduce background interference in the mapped feature matrix, which is contrary to the purpose of oriented detection. Comparing with RoI Pooling, the detection performance of our model with the RRoI Pooling achieve the recall of $98.00 \%$, precision of $95.56 \%$, and F-score of $96.76 \%$ with the RRoI Pooling procedure.

TABLE IV

COMPARISON OF DiFFERENT POOLING METHOdS

\begin{tabular}{cccc}
\hline \hline Module & Precision $(\%)$ & Recall $(\%)$ & $\mathrm{F}(\%)$ \\
\hline RoI Pooling & 71.35 & 89.20 & 79.28 \\
RRoI Pooling & $\mathbf{9 5 . 5 6}$ & $\mathbf{9 8 . 0 0}$ & $\mathbf{9 6 . 7 6}$ \\
\hline \hline
\end{tabular}

\section{3) Effect of the FPN}

The experimental results are shown in Table V. Among them, Res50_b represents the improved version of the residual network with fifty layers. Compared with our method using only an improved residual network, the addition of FPN brings a performance improvement in terms of detection metrics. The FPN merges shallow features and deep features through bottom-up and horizontal connections, enriches the information of the target's features, and improves the multi-scale detection ability of the model.

TABLE V COMPARISON OF DIFFERENT FUSION METHODS

\begin{tabular}{cccc}
\hline \hline Model & Precision $(\%)$ & Recall $(\%)$ & $\mathrm{F}(\%)$ \\
\hline Res50_b & 89.08 & 93.27 & 91.13 \\
Res50_b+FPN & $\mathbf{9 5 . 5 6}$ & $\mathbf{9 8 . 0 0}$ & $\mathbf{9 6 . 7 6}$ \\
\hline \hline
\end{tabular}

\section{F. Experiment Results and Evaluation}

\section{1) Qualitative comparison with Faster R-CNN}

Based on the improved ResNet50 architecture, we conducted a qualitative comparative experiment on Faster R-CNN [22]. The detection results obtained are demonstrated in Fig. 10. From the previous discussion in Section II, the Faster R-CNN cannot effectively separate the tilted double insulator string and the mutually occluded insulator string. Interestingly, the 
This article has been accepted for publication in a future issue of this journal, but has not been fully edited. Content may change prior to final publication. Citation information: DOI10.1109/TII.2021.3123107, IEEE Transactions on Industrial Informatics

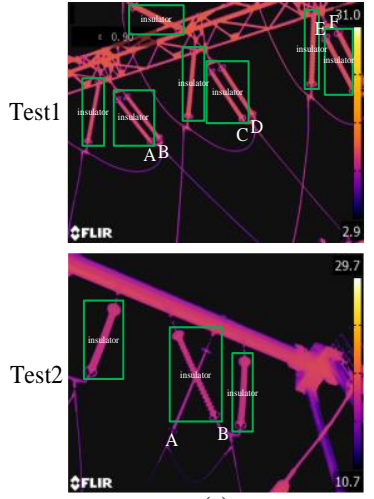

(a)

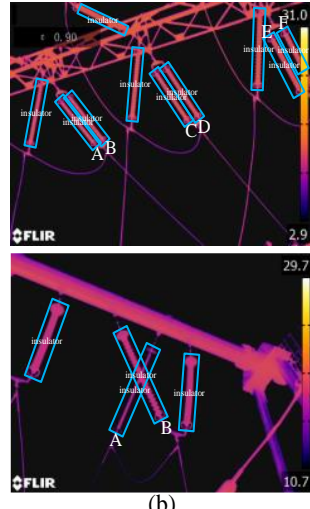

(b)
Fig. 10. The detection result on constructed infrared dataset. (a) Faster R-CNN. (b) our method.

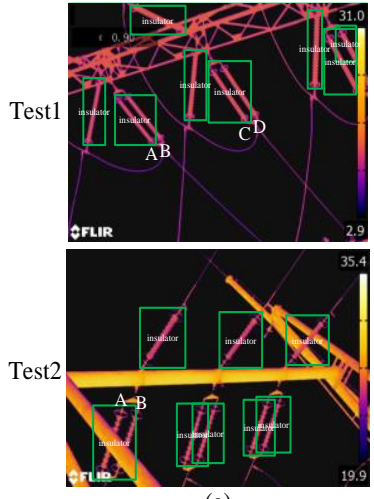

(a)

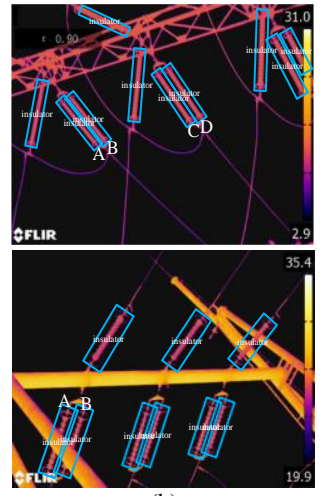

(b)
Fig. 11. The detection result on constructed infrared dataset. (a) SSD. (b) our method.

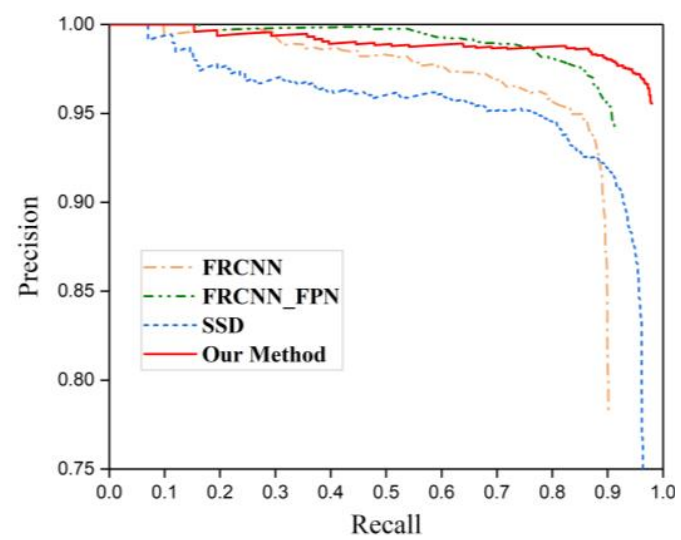

Fig. 12. P-R curves of different detection methods.

TABLE VI

PERFORMANCE COMPARISON OF DIFFERENT DETECTION METHODS

\begin{tabular}{cccccc}
\hline \hline Model(backbone) & Precision(\%) & Recall $(\%)$ & $\mathrm{F}(\%)$ & $\mathrm{AP}_{50}(\%)$ & $\mathrm{FPS}$ \\
\hline SSD(VGG16) & 60.25 & 96.63 & 74.22 & 87.71 & $\mathbf{3 9 . 7}$ \\
FRCNN(Res50_b) & 78.28 & 90.11 & 83.78 & 88.35 & 9.3 \\
FRCFPN(Res50_b) & 94.17 & 91.20 & 92.66 & 92.13 & 8.9 \\
Ours(Res50_b) & $\mathbf{9 5 . 5 6}$ & $\mathbf{9 8 . 0 0}$ & $\mathbf{9 6 . 7 6}$ & $\mathbf{9 5 . 0 8}$ & 6.3 \\
\hline \hline
\end{tabular}

method proposed in this paper considers the tilt and occlusion problem caused by the mechanical properties of insulators and complex filming sites. The detection results are rotating detection boxes, which can minimize background interference and improve the signal-noise ratio. Last but not least, the rotating detection box of its output can effectively separate the contour of the insulator string, which lays a solid foundation for subsequent diagnosis automation task.

\section{2) Comparison experiment with other detection methods}

We also carry out a qualitative comparison experiment with SSD [19] model, and the test results are shown in Fig. 11. It can be seen from Fig. 11 that the SSD has a good detection effect in single insulator strings, but the detection effect is not so good in the detection of an inclined twin insulator strings. As shown in Fig. 11, the insulators B and D of the twin insulator string in Test1 were not detected. In addition, due to background interference, insulators B in Test 2 was not detected. Compared with the SSD, the method proposed in this paper reflects the actual shape of insulators and greatly reduces background interference. It can not only separate densely distributed insulator strings, but also detect insulator strings shielded by an angle iron (B in Test2).

Table VI summarizes and evaluates the detection performance of four different methods. Among them, FRCFPN represents the traditional non-oriented detection method that combines Faster R-CNN and feature pyramid network. It can be seen that our method exceeds the traditional non-oriented detection methods in terms of precision, recall, and average precision. Specifically, the recall rate and precision reached $95.56 \%$ and $98.00 \%$, respectively. The $\mathrm{AP}_{50}$ achieves $95.08 \%$ and the F-score was $96.76 \%$. Fig. 12 shows the P-R curves of different methods. As we can see, P-R curve of our method is higher than those of Faster R-CNN, and SSD. The performance improvement is mainly attributed to the collaborative work of the multiple modules in the oriented detection task. Table VI also comparatively lists the detection time required by different detection methods. Since our method will generate more prior boxes than Faster R-CNN in the test phase, these candidate regions will take a long time when NMS is used. This will result in the final detection speed slightly lower than the traditional target detection algorithm.

\section{V.CONCLUSION}

This paper proposes a high-precision oriented detection method for insulator infrared images based on improved faster R-CNN. The original network model has been improved in five aspects: rectangular box representation, feature extraction, candidate box generation, anchor design, and feature alignment. In terms of rectangular box representation, the oriented detection rectangular box with an angle can effectively reflect the shape information and angle information of the object, and at the same time reduce the risk of missed detection caused by NMS deleting the detection box; in terms of feature extraction, the feature extraction network that combines the improved ResNet and FPN can make full use of feature information, reduce network calculations, and better complete the fusion of multi-scale feature information; in terms of candidate box generation, RRPN can generate high-quality rotating candidate regions containing potential objects; in terms of anchor design, the design strategy combining K-means++ and histogram statistics can not only generate anchors that are closer to ground truth box, but also the model can better distinguish objects with 
similar background; in terms of feature alignment, the rotating interest pooling can more effectively match the candidate region with the feature matrix, retain the features that are useful for the detection task, and suppress the background and features that are not helpful to the task. The ablation experiment on the constructed insulator dataset verifies the effectiveness of the improved scheme. Subsequently, comparative experiments with a variety of mainstream detection methods further prove the advantages of the method can effectively solve the defects of the horizontal box detection method.

However, because the model needs to generate a large number of prior boxes, it will take more time and cost, so the proposed high-precision detection method is not suitable for real-time detection. Because of this, one of our future tasks will combine the proposed method with the temperature information of the infrared images to realize the refined fault diagnosis of insulators.

\section{REFERENCES}

[1] Y. Tu, B. Gong, C. Wang, et al., "Effect of moisture on temperature rise of composite insulators operating in power system," IEEE Transactions on Dielectrics and Electrical Insulation, vol. 22, no. 4, pp. 2207-2213, Aug. 2015.

[2] H. Zheng, Y. Sun, X. Liu, et al., "Infrared image detection of substation insulators using an improved fusion single shot multibox detector," IEEE Transactions on Power Delivery, early access, 2020, doi: 10.1109/TPWRD.2020.3038880

[3] Y. Liu and B. Du, "Recurrent plot analysis of leakage current in dynamic drop test for hydrophobicity evaluation of silicone rubber insulator," IEEE Transactions on Power Delivery, vol. 28, no. 4, pp. 1996-2003, Oct. 2013.

[4] Z. Zhao, G. Xu, and Y. Qi, "Representation of binary feature pooling for detection of insulator strings in infrared images," IEEE Transactions on Dielectrics and Electrical Insulation, vol. 23, no. 5, pp. 2858-2866, Oct. 2016

[5] M. Jadin, S. Taib, and K. Ghazali, "Finding region of interest in the infrared image of electrical installation," Infrared Physics \& Technology, vol. 71, pp. 329-338, Jul. 2015.

[6] Z. Zhao, X. Fan, G. Xu, et al., "Aggregating deep convolutional feature maps for insulator detection in infrared images," IEEE Access, vol. 5, pp. 21831-21839, Sept. 2017.

[7] W. L. Chan, A. So, and L. L. Lai, "3-Dimensional thermal imaging for power equipment monitoring," IEE Proceedings - Generation, Transmission and Distribution, vol. 147, no. 6, pp. 355-360, Nov. 2000.

[8] A. Alhassan, X. Zhang, H. Shen, et al., "Power transmission line inspection robots: A review, trends and challenges for future research," International Journal of Electrical Power \& Energy Systems, vol. 118, p. 105862, Jun. 2020.

[9] S. Lu, Y. Zhang, and J. Su, "Mobile robot for power substation inspection a survey," IEEE/CAA Journal of Automatica Sinica, pp. 1-18, Jan. 2017.

[10] Y. LeCun, Y. Bengio, and G. Hinton, "Deep learning," Nature, vol. 521, no. 7553, pp. 436-444, May. 2015.

[11] R. Girshick, J. DonahueRoss, T. Darrell et al., "Region-based convolutional networks for accurate object detection and segmentation," IEEE Transactions on Pattern Analysis and Machine Intelligence., vol. 38, no. 1, pp. 142-158, May, 2015

[12] R. Girshick, "Fast r-cnn," arXiv preprint arXiv: 1504.08083, 2015.

[13] L. Yang, J. Fan, Y. Liu, et al., "A review on state-of-the-art power line inspection techniques," IEEE Transactions on Instrumentation and Measurement, vol. 69, no. 12, pp. 9350-9365, Oct. 2020.

[14] Q. Wu and J. An, "A texture segmentation algorithm based on PCA and global minimization active contour model for aerial insulator images," IEEE Transactions on Geoscience and Remote Sensing., vol. 52, no. 6, pp. 3613-3626, Jun. 2014.

[15] X. Yao, L. Liu, Z. Li, et al., "Identification method of railway insulator based on edge features," MS\&E., vol. 394, no. 3, 2018.

[16] Z. Zhao, N. Liu, and L. Wang, "Localization of multiple insulators by orientation angle detection and binary shape prior knowledge," IEEE
Transactions on Dielectrics and Electrical Insulation., vol. 22, no. 6, pp. 3421-3428, Dec. 2015.

[17] T. Jabid, T. Ahsan. "Insulator detection and defect classification using rotation invariant local oriented pattern," Int. J. Adv. Comput. Sci. Appl., vol. 9, no. 2, pp. 265-272, 2018.

[18] B. Wang, F. Ma, L. Ge, H. Ma, H. Wang, and M. A. Mohamed, "Icing-EdgeNet: a pruning lightweight edge intelligent method of discriminative driving channel for ice thickness of transmission lines," IEEE Transactions on Instrumentation and Measurement., vol. 70, Aug. 2020

[19] W. Liu, D. Anguelov, D. Erhan, et al., "SSD: Single Shot MultiBox Detector," in European Conference on Computer Vision, Amsterdam, The Netherlands, Oct. 2016, pp. 21-37.

[20] X. Tao, D. Zhang, Z. Wang, X. Liu, H. Zhang, and D. Xu, "Detection of power line insulator defects using aerial images analyzed with convolutional neural networks," IEEE Transactions on Systems, Man, and Cybernetics: Systems., vol. 50, no. 4, pp. 1486-1498, Apr. 2020.

[21] Z. Zhao, Z. Zhen, L. Zhang, Y. Qi, Y. Kong, and K. Zhang, "Insulator detection method in inspection image based on improved Faster R-CNN," Energies., vol. 12, no. 7, pp. 1204-1218, Mar. 2019.

[22] S. Ren, K. He, R. Girshick, and J. Sun, "Faster R-CNN: Towards real-time object detection with region proposal networks," arXiv preprint arXiv: 1506.01497, 2016.

[23] Y. Liu, X. Ji, S. Pei, Z. Ma, G. Zhang, Y. Lin, and Y. Chen, "Research on automatic location and recognition of insulators in substation based on YOLOv3," High Voltage., vol. 5, no. 1, pp. 62-68, Mar. 2020.

[24] L. Liu, Z. Pan, and B. Lei, "Learning a rotation invariant detector with rotatable bounding box," arXiv preprint arXiv:1711.09405, 2017.

[25] T. Tang, S. Zhou, Z. Deng, et al., "Arbitrary-oriented vehicle detection in aerial imagery with single convolutional neural networks," Remote Sensing., vol. 9, no. 11, p. 1170, Nov. 2017.

[26] C. Li, Q. Zhang, W. Chen, et al., "Insulator orientation detection based on deep learning," Journal of Electronics and Information Technology, vol. 42, no. 4, pp. 1033-1040, 2020.

[27] X. Gong, Q. Yao, M. Wang, and Y. Lin, "A deep learning approach for oriented electrical equipment detection in thermal images," IEEE Access., vol. 6, pp. 41590-41597, Jul. 2018.

[28] Y. Lin, M. Wang, C. Gu, et al., "A cascaded spatial transformer network for oriented equipment detection in thermal images,"2nd IEEE Conference on Energy Internet and Energy System Integration (EI2), 2018, pp. 1-5.

[29] K. He, X. Zhang, S. Ren, et al., "Deep residual learning for image recognition," in Proceedings of the IEEE Conference on Computer Vision and Pattern Recognition, Las Vegas, USA, Jun. 2016, pp. 770-778.

[30] T. Lin, P. Dollár, R. Girshick, et al., "Feature Pyramid Networks for Object Detection," in Proceedings of the IEEE Conference on Computer Vision and Pattern Recognition, Honolulu, USA, Jul, 2016, pp. 2117-2125

[31] M. Everingham, L. Gool, C. Williams, et al., "The Pascal Visual Object Classes (VOC) Challenge," International Journal of Computer Vision, vol. 88 , no. 2 , pp. 303-338, Sept. 2009.

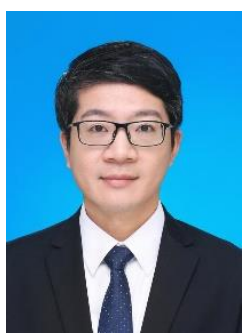

Hanbo Zheng (Senior Member, IEEE) was born in Henan, China. He received the M.Sc. and Ph.D. degrees in electrical engineering from Chongqing University, Chongqing, China, in 2009 and 2012, respectively. From 2010 to 2011, he was a Visiting Scholar with the Department of Electrical Engineering and Computer Science, University of Tennessee, Knoxville, USA. From 2012 to 2018, he worked at State Grid Henan Electric Power Research Institute, where he was a Senior Engineer in electrical engineering. In 2018, he joined the School of Electrical Engineering of Guangxi University, and he is now an Associate Professor at there.

His main research interests are in the areas of condition assessment and intelligent diagnosis for electrical equipment, electrical insulation materials, and smart distribution grid. 
This article has been accepted for publication in a future issue of this journal, but has not been fully edited. Content may change prior to final publication. Citation information: DOI10.1109/TII.2021.3123107, IEEE Transactions on Industrial Informatics

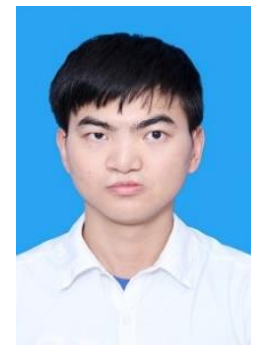

Yang Liu received the M.S. degrees in electrical engineering from Guangxi University, Nanning, China, in 2021. His main research field is power transformer condition assessment and image recognition.

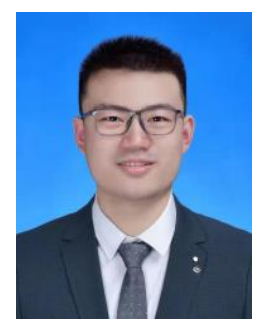

Yonghui Sun received the B.S. degree in electrical engineering and automation from Northeast Electric Power University, Jilin, China, in 2019. He is currently pursuing the M.E. degree in electrical engineering with the School of Electrical Engineering, Guangxi University, Nanning, China. His main research field is intelligent diagnosis and image recognition of electrical equipment.

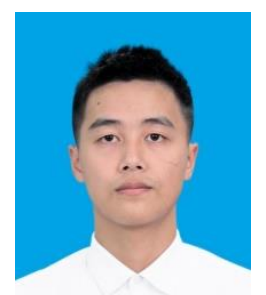

Jinheng Li received the M.S. degrees in electrical engineering from Guangxi University, Nanning, China, in 2021 .

He is currently working toward the Ph.D. degree in electrical engineering with the Electrical Engineering Department, Guangxi University. His current research interest focuses on condition monitoring of power equipment.

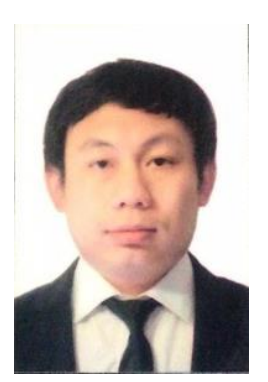

Zhen Shi received the Bachelor degree in electrical engineering in 2010 from Shanghai University of Electric Power. He was studying abroad in the University of Strathclyde UK, where he received the MSc and PhD degrees in 2011 and 2018. He joined Guangxi University in 2018 as a Post doctor of School of Electrical Engineering. His research interests are mainly in the area of advanced partial discharge detection technologies, data analysis of partial discharge detection, and the online evaluation of electrical equipment insulation.

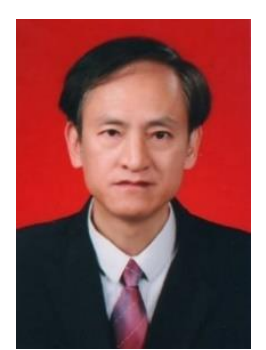

Chaohai Zhang Received the B.A., M.S., and Ph.D. degrees from the Harbin Institute of Technology, Harbin, China, Navy Aeronautical Engineering Academy, Yantai, China, and Hong Kong Polytechnic University, Hong Kong, in 1985, 1988, and 2002, respectively, all in electrical engineering.

$\mathrm{He}$ worked as a JSPS Research Fellow with Kumamoto University, Kumamoto, Japan, and the Chief Research Scientist with State Grid Electric Power Research Institute, Wuhan, China. $\mathrm{He}$ is currently an Adjunct Professor with Guangxi University, Nanning, China. His current research interests are mainly in high voltage engineering, electrical discharges, pulsed power and plasma application, renewable energy and the condition monitoring, diagnosis, and management of electric power equipment.

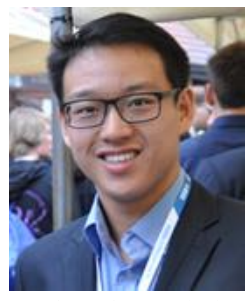

Chun Sing Lai (Senior Member, IEEE) received the B.Eng. (First Class Hons.) in electrical and electronic engineering from Brunel University London, London, UK, in 2013, and the D.Phil. degree in engineering science from the University of Oxford, Oxford, UK, in 2019.

He is currently a Lecturer with the Department of Electronic and Electrical Engineering, Brunel University London. From 2018 to 2020, he was an UK Engineering and Physical Sciences Research Council Research Fellow with the School of Civil Engineering, University of Leeds, Leeds, UK. His current research interests are in power system optimization and data analytics.

Dr. Lai was both the Publications Co-Chair for 2020 and 2021 IEEE International Smart Cities Conferences. He is the Vice-Chair of the IEEE Smart Cities Publications Committee and Associate Editor for IET Energy Conversion and Economics. He is the Working Group Chair for IEEE P2814 Standard, and the Chair of the IEEE SMC Intelligent Power and Energy Systems Technical Committee. He is an IET Member and a Chartered Engineer.

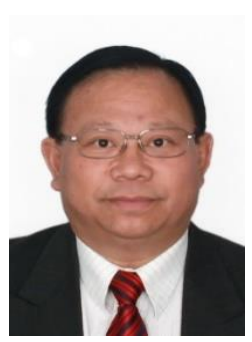

Loi Lei Lai (Life Fellow, IEEE) received the B.Sc. (First Class Hons.), Ph.D., and D.Sc. degrees in electrical and electronic engineering from the University of Aston, Birmingham, UK, and City, University of London, London, UK, in 1980, 1984, and 2005, respectively.

Professor Lai is currently a University Distinguished Professor with Guangdong University of Technology, Guangzhou, China. He was a Pao Yue Kong Chair Professor with Zhejiang University, Hangzhou, China, and the Professor and Chair of Electrical Engineering with City, University of London. His current research areas are in smart cities and smart grid. Professor Lai was awarded an IEEE Third Millennium Medal, the IEEE Power and Energy Society (IEEE/PES) UKRI Power Chapter Outstanding Engineer Award in 2000, the IEEE/PES Energy Development and Power Generation Committee Prize Paper in 2006 and 2009, the IEEE/SMCS Outstanding Contribution Award in 2013 and 2014, the Most Active Technical Committee Award in 2016, and his research team has received a Best Paper Award in the IEEE International Smart Cities Conference in October 2020.

Professor Lai is an Associate Editor of the IEEE Transactions on Systems, Man, and Cybernetics: Systems, Editor-in-Chief of the IEEE Smart Cities Newsletter, a member of the IEEE Smart Cities Steering Committee and the Chair of the IEEE Systems, Man, and Cybernetics Society (IEEE/SMCS) Standards Committee. He was a member of the IEEE Smart Grid Steering Committee; the Director of Research and Development Center, State Grid Energy Research Institute, China; a Vice President for Membership and Student Activities with IEEE/SMCS; and a Fellow Committee Evaluator for the IEEE Industrial Electronics Society. He is a Fellow of IET. 